\title{
Escape-capture bigeminy in rate dependent (phase 3) block of atrioventricular conduction
}

\author{
J. Tenczer, L. Littmann, and T. Fenyvesi \\ From the 3rd Department of Medicine, Semmelweis University of Medicine, Budapest, Hungary
}

A specific, hitherto unpublished mechanism of escape-capture bigeminy based on rate dependent (phase 3) block of atrioventricular conduction is described.

Escape-capture bigeminy is a bigeminal rhythm in which each escape beat is followed by a conducted beat. Bradley and Marriott (1958) were the first to describe this interesting electrocardiographic pattern in a case of sinoatrial block. Schamroth and Dubb (1965) reported cases of escape-capture bigeminy resulting from sinoatrial block, atrioventricular block, and a second degree Wenckebach type atrioventricular block ending in an atrial echo beat. A fourth mechanism was published by Dubb and Schamroth (1975) in which escapecapture bigeminy was caused by blocked atrial extrasystoles. We present a case of escape-capture bigeminy caused by a specific, hitherto unpublished mechanism.

\section{Case report}

A 73-year-old man with coronary heart disease was admitted with a history of retrosternal chest pain. No myocardial infarction could be proved. He received no cardioactive medication.

Electrocardiogram on admission showed sinus arrhythmia with left bundle-branch block and atrioventricular dissociation caused by advanced atrioventricular block. For a short period constant ratio of block with bigeminy could be seen after which the advanced atrioventricular block with varying ratio of block returned. One week after admission the atrioventricular conduction disturbance disappeared.

Fig. 1 shows sinus arrhythmia, second degree atrioventricular block, with varying ratio of block. Most of the sinus impulses are not transmitted to the ventricles. An escape rhythm is interrupted by sinus beats transmitted with normal or prolonged $P R$ intervals. If a $P$ wave follows the escape $Q R S$ complex closely, the next sinus impulse is con- ducted to the ventricles (5th, 12th, and 17th $P$ waves). If the $P$ wave falls just before or is concealed within the QRS complex of the escape beat (3rd, 8th, 10th, and 20th $P$ waves), the following $P$ is not conducted to the ventricles. Fig. 2 shows an escape-capture bigeminy pattern. At an altered sinus and escape frequency the preceding $P$ wave of the conducted sinus impulse follows closely (3rd and 6th P waves) or is concealed within (9th and 12th $P$ waves) the QRS complex of the escape.

\section{Discussion}

Frequency dependency of the impulse conducting structures of the heart is a proved electrophysiological phenomenon (Mendez, Gruhzit, and Moe, 1956). The functional behaviour of the atrioventricular junction and His-Purkinje system both in the antegrade and retrograde direction has been extensively studied in clinical electrophysiology (Akhtar et al., 1975). Our case is a specific manifestation of an atrioventricular block based on frequency dependency of the atrioventricular conduction (Moe, Childers, and Merideth, 1968). The two special characteristics of the case are the following: (1) The electrocardiographic pattern was induced by a bidirectional stimulation of the blocking zone. (2) At a given ratio of the sinus and escape frequency escape-capture bigeminy occurred.

It is apparent from the ladder diagrams that the atrioventricular conduction of the sinus beats is not accidental but follows the rule of rate dependency. Atrioventricular conduction can only occur if two consecutive stimuli arriving at the blocking zone reach a critical time interval. This time interval must exceed the effective refractory period (ERP) of the region of block. If the interval be- 


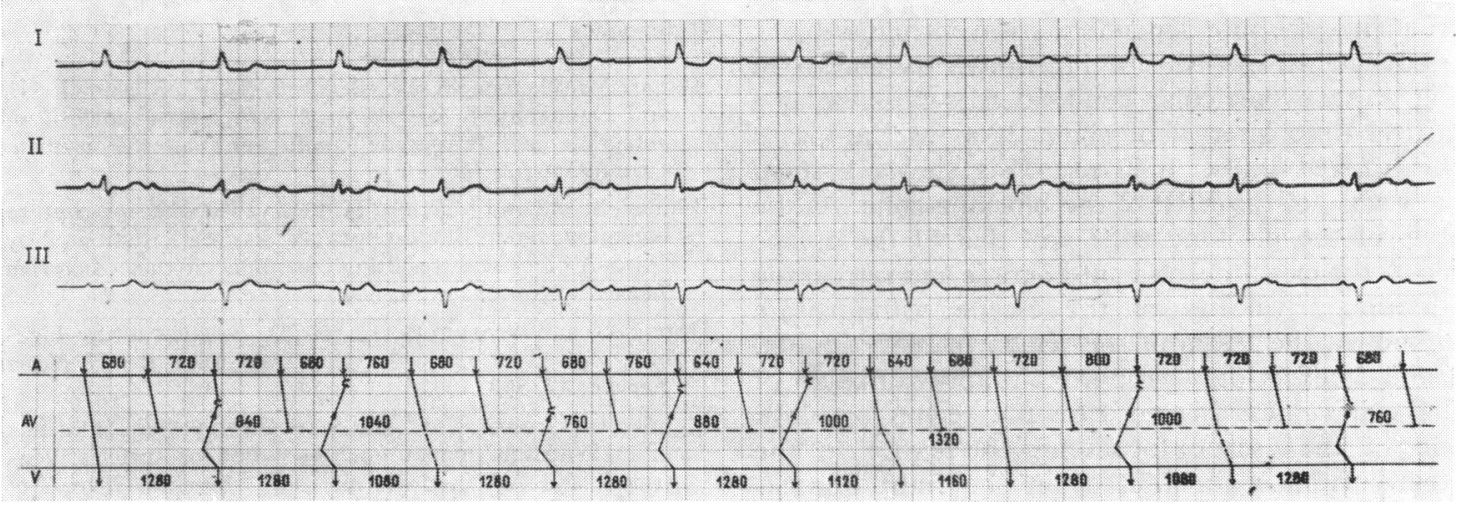

FIG. 1 Rate dependent block of the atrioventricular conduction. Dotted line represents the blocking zone.

tween two consecutive impulses is below the critical time, the second impulse is blocked. The interval between two sinus impulses is always shorter than the ERP of the blocking zone. Had the blocking zone not been stimulated by any other stimulus, regular 2:1 atrioventricular block would have occurred. In our case the region of block is depolarized by stimuli of escape origin too, changing the sequence of stimulation and the atrioventricular blocking ratio. The propagation of an atrial impulse to the ventricles becomes-in a peculiar waystrictly dependent on a proper time relation of an atrial impulse to the discharge of the escape pacemaker. The escape impulse depolarizes the blocking zone before the corresponding sinus impulse could have reached it. The concealed retrograde conduction of the escape beat prevents this sinus impulse from reaching and stimulating the blocking zone. Thus the time interval of two consecutive stimuli reaching and activating the blocking zone will exceed that of the corresponding PP interval. If the $P$ wave falls just before or into the escape QRS complex, the increase will be small. If the escape QRS is followed by the $P$ wave, this increase will be greater. In case of sinus arrhythmia the increase will also be influenced by the actual PP interval. When the critical simulusstimulus interval in the blocking zone is reached, atrioventricular conduction will occur. The conduction of the 15th sinus impulse (Fig. 1) to the ventricles is somewhat different. After the prolonged atrioventricular conduction of the 13th $P$ depolarization of the escape region is delayed, and the 15th $P$ can reach the blocking zone before the next escape impulse. It seems likely that this complex picture can well be interpreted on the basis of rate dependency of atrioventricular conduction.

The basic mechanism of the escape-capture bigeminy shown in Fig. 2 appears to be the same. The time intervals between two stimuli arriving at the blocking zone are similarly increased by the escape beats. An altered sinus and escape frequency produced a situation where every third $\mathbf{P}$ wave was related to the escape $Q R S$ as described above. The augmented time interval of two stimuli at the blocking zone produced by the escape stimulus always exceeded the critical time interval. Thus, at this given ratio of the altered sinus and escape

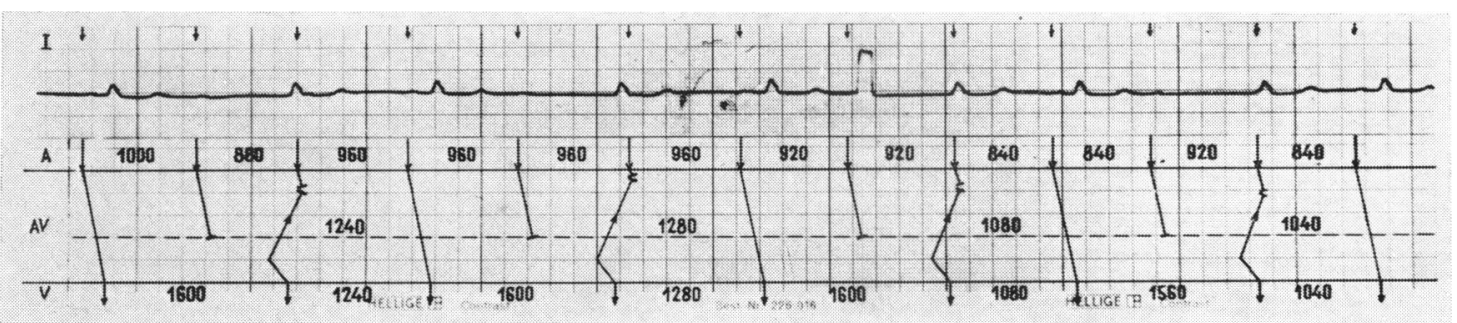

FIG. 2 Escape-capture bigeminy. 
frequency the rate dependent atrioventricular block produced escape-capture bigeminy. As shown in Fig. 1 atrioventricular conduction is possible at a critical time interval of about $1000 \mathrm{~ms}$; in Fig. 2 this proved to be $1040 \mathrm{~ms}$. This shows a small variability of the ERP of the blocking zone. As the level of the blocking zone and that of the escape focus, the exit time, and anterograde and retrograde conduction velocity of the escape stimulus are unknown, the ERPs of the region of block in Fig. 1 and 2 reflect only proportional approximation of the real values. In our case the region of block and of the escape focus may be either in the atrioventricular junction or in the right bundlebranch.

Escape-capture bigeminy in frequency dependent atrioventricular block differs thoroughly from escape-capture bigeminy in stable second degree atrioventricular block. In the latter there is no cause-and-effect relation between the escape and conducted sinus impulse. In our case with rate dependent block of the atrioventricular conduction the changes produced by the escape impulse created the condition for the next sinus impulse to be transmitted.

\section{References}

Akhtar, M., Damato, A. N., Batsford, W. P., Ruskin, J. N., and Ogunkelu, J. B. (1975). A comparative analysis of antegrade and retrograde conduction patterns in man. Circulation, 52, 766.

Bradley, S. M., and Marriott, H. J. L. (1958). Escape-capture bigeminy: report of a case of $\mathrm{A}-\mathrm{V}$ dissociation initiated by 2:1 S-A block with resulting bigeminal rhythm. American fournal of Cardiology, 1, 640.

Dubb, A., and Schamroth, L. (1975). Escape-capture bigeminy caused by blocked atrial extrasystoles. British Heart fournal, 37, 890.

Mendez, C., Gruhzit, C. C., and Moe, G. K. (1956). Influence of cycle length upon refractory period of auricles, ventricles, and A-V node in the dog. American fournal of Physiology, 184, 287.

Moe, G. K., Childers, R. W., and Merideth, J. (1968). An appraisal of 'supernormal' A-V conduction. Circulation, 38, 5.

Schamroth, L., and Dubb, A. (1965). Escape-capture bigeminy: mechanisms in S-A block, A-V block, and reversed reciprocal rhythm. British Heart fournal, 27, 667.

Requests for reprints to Dr. J. Tenczer, 3rd Department of Medicine, Semmelweis University of Medicine, Mezö Imre ut 17, Budapest, Hungary. 